\title{
Probing Magnetic Defects in Ultra-Scaled Nanowires with Optically Detected Spin Resonance in Nitrogen-Vacancy Center in Diamond
}

Umberto Celano $^{1,2, *}$, Hai Zhong ${ }^{3}$, Florin Ciubotaru ${ }^{1}$, Laurentiu Stoleriu ${ }^{4}$, Alexander Stark ${ }^{3}$, Peter Rickhaus ${ }^{3}$, Felipe Fávaro de Oliveira ${ }^{3}$, Mathieu Munsch ${ }^{3}$, Paola Favia ${ }^{1}$, Maxim Korytov $^{1}$, Patricia Van Marcke ${ }^{1}$, Patrick Maletinsky ${ }^{3,5}$, Christoph Adelmann ${ }^{1}$ and Paul van der Heide ${ }^{1}$

1 imec, Kapeldreef 75, 3001, Leuven, Belgium.

2 Faculty of Science and Technology and MESA+ Institute for Nanotechnology, University of Twente, Enschede, The Netherlands

3 Qnami AG, Hofackerstrasse 40B, CH-4132 Muttenz, Switzerland

4 Department of Physics, Alexandru Ioan Cuza University, Bd. Carol I 11, 700506 Iasi, Romania

5 Department of Physics, University of Basel, Klingelbergstrasse 82, Basel CH-4056, Switzerland

* Correspondence and requests for materials should be addressed to Umberto Celano (email: umberto.celano@imec.be).

Supporting Information Fig. S1: Fabrication of $\mathrm{CoFeB}$ nanowires

To fabricate the structures of this work we use the concept of gate-spacers from CMOS technology. Here, $40 \mathrm{~nm}$ thick $\mathrm{SiO}_{2}$ lines are patterned first using a $300 \mathrm{~nm}$ pitch. The $\mathrm{SiO}_{2}$ deposition is followed by the conformal deposition of CoFeB (30 nm thick), and lastly by the etching of $\mathrm{CoFeB}$ using ion beam etching (IBE) to leave a thin $\mathrm{CoFeB}$ layer only in correspondence of the sidewalls of the $\mathrm{SiO}_{2}$ lines.
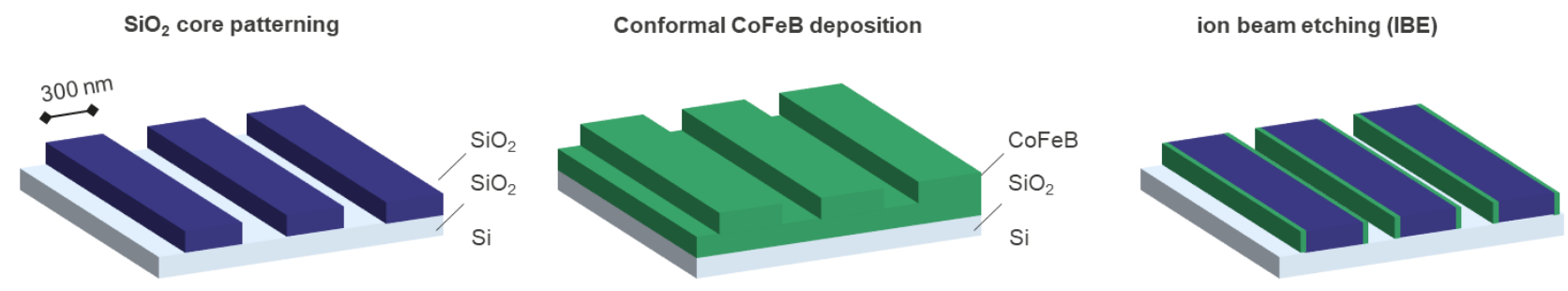

Figure S1. Fabrication steps of the sub-10 nm CoFeB nanowires. 
Supporting Information Fig. S2: Structural analysis of CoFeB nanowires

Scanning electron microscopy and transmission electron microscopy are used for the structural characterization of the CoFeB NWs. In $\mathbf{a}$ and $\mathbf{b}$ we monitor the quality of the process using top view SEM, while in $\mathbf{c}$ and $\mathbf{d}$, TEM is used in cross section i.e., Titan operating at $200 \mathrm{kV}$. The analysis with TEM along the wire (e) indicates that the $\mathrm{CoFeB}$ is continuous and amorphous with no appearance of crystallites. 
a

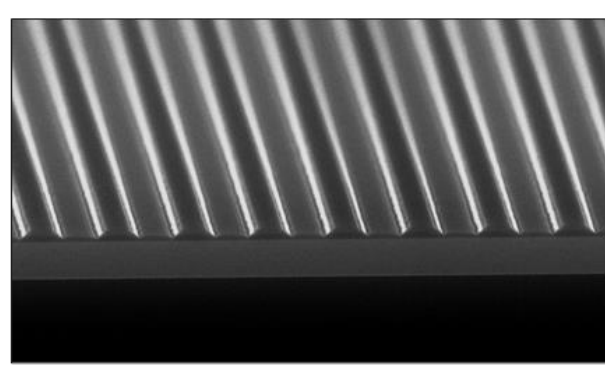

b

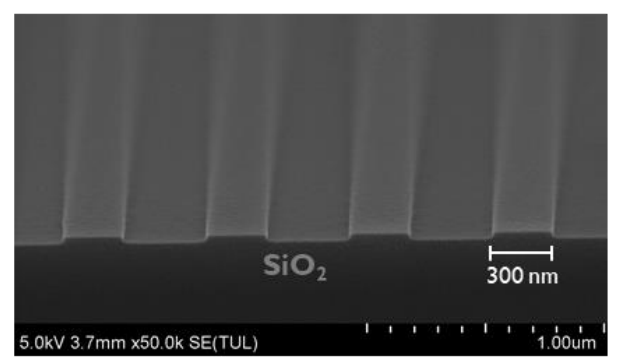

C

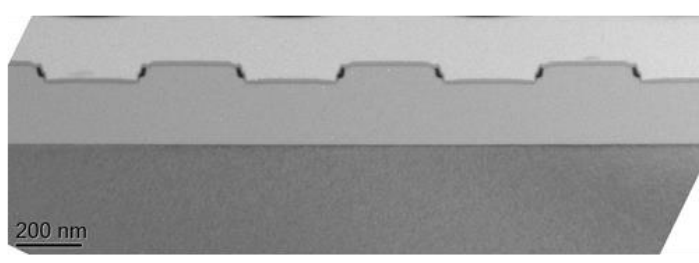

d

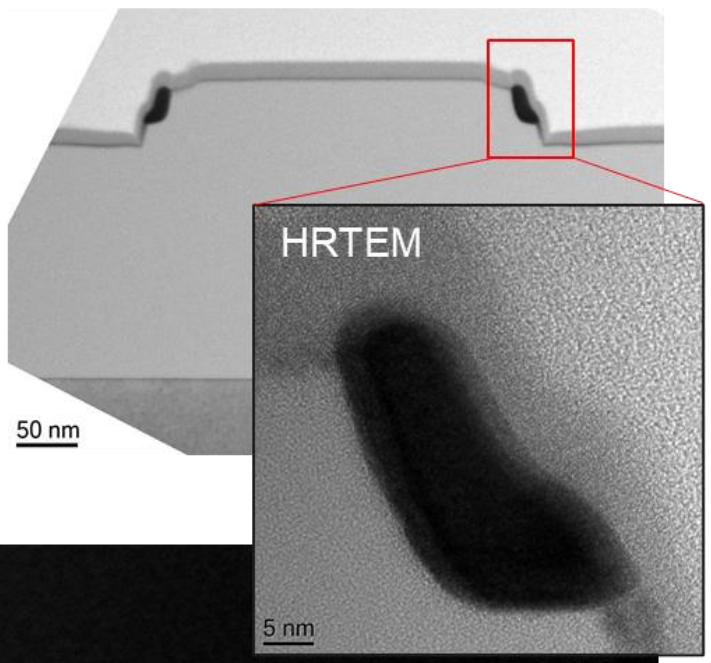

HRTEM

e

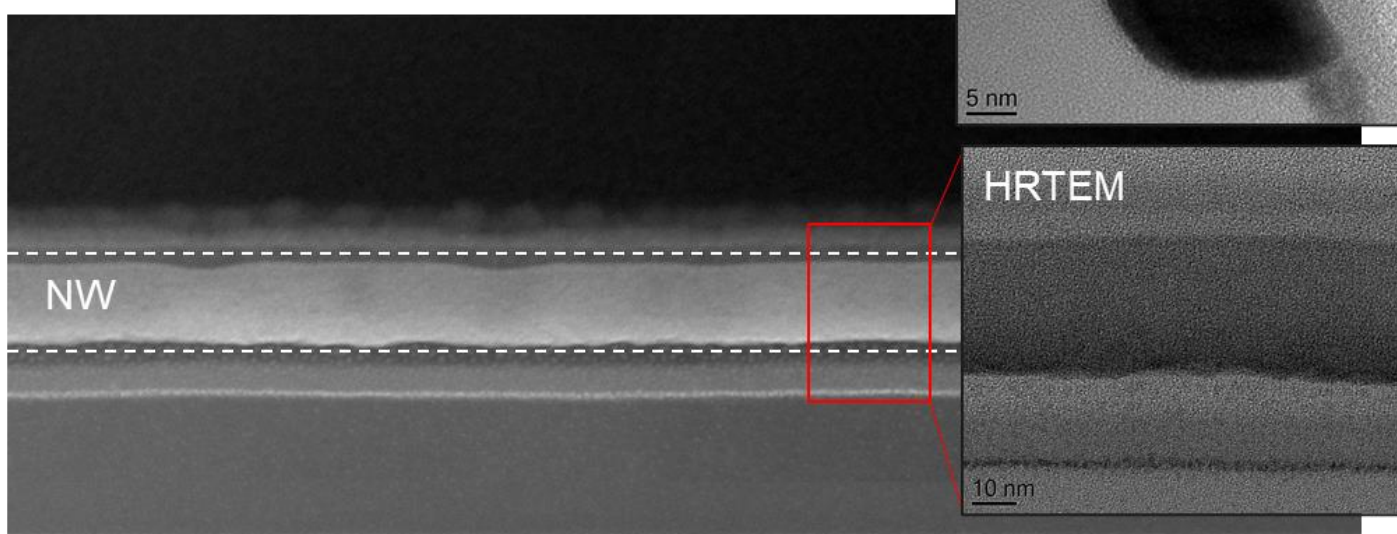

\section{$100 \mathrm{~nm}$}

Figure S2. Structural characterization of CoFeB nanowires with SEM and TEM.

Supporting Information Fig. S3: First Order Reversal Curves analysis

A magnetization loop describes the magnetization behavior as a function of the external applied field. The saturation magnetization denotes the value for which no further changes in the magnetization occurs while increasing the external magnetic field.

For a system consisting of many magnetic entities, a First Order Reversal Curve is obtained by saturating the system in one direction followed by the application of a reversal magnetic field with a certain value $\mathrm{H}_{\mathrm{r}}$ and then measuring $\mathrm{M}(\mathrm{H})$ by increasing the field back to initial saturation (see Fig. S3.). 
The FORC distribution is obtained by second order mixed derivative $\mathrm{M}\left(\mathrm{H}, \mathrm{H}_{\mathrm{r}}\right)$ :

$$
\rho=-\frac{1}{2} \frac{\partial^{2} M}{\partial H \partial H_{r}}
$$

and can be represented in either $\left(\mathrm{H}, \mathrm{H}_{\mathrm{r}}\right)$ coordinates or in $\left(\mathrm{H}_{\mathrm{c}}, \mathrm{H}_{\mathrm{i}}\right)$ coordinates (switching and interaction field, respectively) using the following transforming relations:

$$
H_{c}=\frac{H-H_{R}}{\sqrt{2}} \quad H_{i}=\frac{H_{R}-H}{\sqrt{2}}
$$

The $\left(\mathrm{H}_{\mathrm{c}}, \mathrm{H}_{\mathrm{i}}\right)$ coordinate system is usually preferred since it provides a better image with direct physical insight of the magnetic properties of the investigated system. The FORC diagram provides in fact the statistical distribution of the switching fields of the magnetic entities within the sample, as well as a measure of the interaction (e.g. via dipolar coupling) between them.

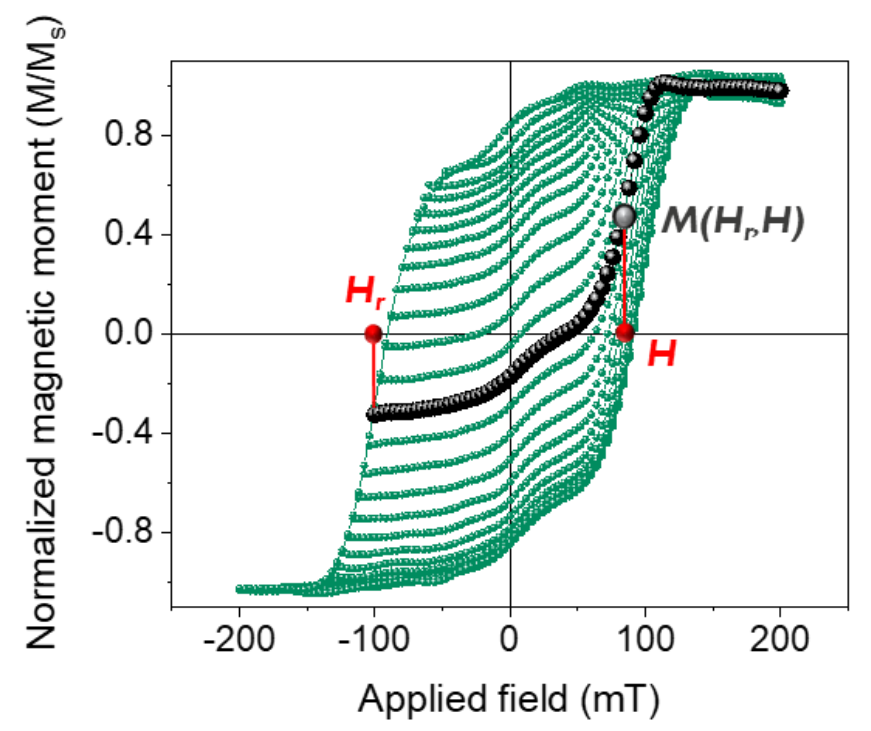

Figure S3. Hysteresis loop and a set of First Order Reversal Curves for magnetic fields applied along the nanowires.

Supporting Information Fig. S4: NV-to-sample distance calibration The NV-to-sample (or NV fly height) $d$ between the NV spin sensor and the sample surface (cf. Fig. 1S) was inferred by mapping the stray magnetic field generated above the upward (downwards) edge of a uniformly magnetized ferromagnetic thin film strip, made of e.g., $\mathrm{CoFeB}$. The measured $B_{\mathrm{NV}}$ profile was recorded while scanning the NV center across the two edges of $20 \mu \mathrm{m}$ wide $[\mathrm{Ta} / \mathrm{CoFeB}(\sim 1 \mathrm{~nm}) / \mathrm{MgO} / \mathrm{Ta}]$ strip as shown in Fig. S4. The distance $d$ 
is then extracted by fitting the experimental data, following the methods described in detail in [1]. To numerically analyze the NV signal $B_{\mathrm{NV}}$ across either the up-step or down-step edge, we use the approximation of $d \gg t$, which is true here for the calibration sample we used. In this case, the stray fields along the $x$ and $z$ axis as depicted in Fig. S4 can be calculated by[1],

$$
\begin{gathered}
B_{x}(x)=\frac{\mu_{0} I_{s}}{2 \pi} \frac{d}{x^{2}+d^{2}} \\
B_{z}(x)=-\frac{\mu_{0} I_{s}}{2 \pi} \frac{x}{x^{2}+d^{2}}
\end{gathered}
$$

where the CoFeB thickness was characterized to be about $0.8 \mathrm{~nm}$, with estimated thin film magnetization of $M \mathrm{~s}=1.2 \times 10^{6} \mathrm{~A} / \mathrm{m}$, generating an effective surface density of magnetic moments of $I_{\mathrm{S}}=M_{\mathrm{S}} \cdot t=9 \times 10^{-4} \mathrm{~A}$. The NV signal $B_{\mathrm{NV}}$ can therefore calculated by taking the scale product (cf. Eqn. S1) between the NV directional unity vector $t_{\mathrm{NV}}=[\sin \theta \cos \phi, \sin \theta \sin \phi$, $\cos \theta]$ and the magnetic fields at the position $x$ via i.e., $\boldsymbol{B}_{\mathrm{NV}}=\left[\mathbf{B}_{x}, B_{y}, \boldsymbol{B}_{z}\right] \cdot \boldsymbol{t}_{\mathrm{NV}}$. The $\mathrm{NV}$ orientation angle $(\theta, \phi)$ of the commercial diamond NV tip (Quantilever MX, Qnami) used in this study has nominal factory characterized values of $\left(\theta_{0}, \phi_{0}\right)=\left(55^{\circ}, 270^{\circ}\right)$ in the laboratory coordinator defined in the main text. Together with the NV-to-sample distance $d,(\theta, \phi)$ are used as free fitting parameters in our non-linear fittings using $\left(\theta_{0}, \phi_{0}\right)$ as initial parameters. The fitting was done in a collective way for both up-step and down-step sides, giving the fitted parameters of $d_{\mathrm{NV}}=59.7 \pm 1.8 \mathrm{~nm}$, and $\theta=57.1^{\circ} \pm 2.5^{\circ}, \phi=270.3^{\circ} \pm 0.9^{\circ}$. In Fig. S4, the result of the fitting is indicated as the red lines, showing good agreement with the measured data (blue scatters). The error bars are due to the statistical fluctuations of multiple measurements. 


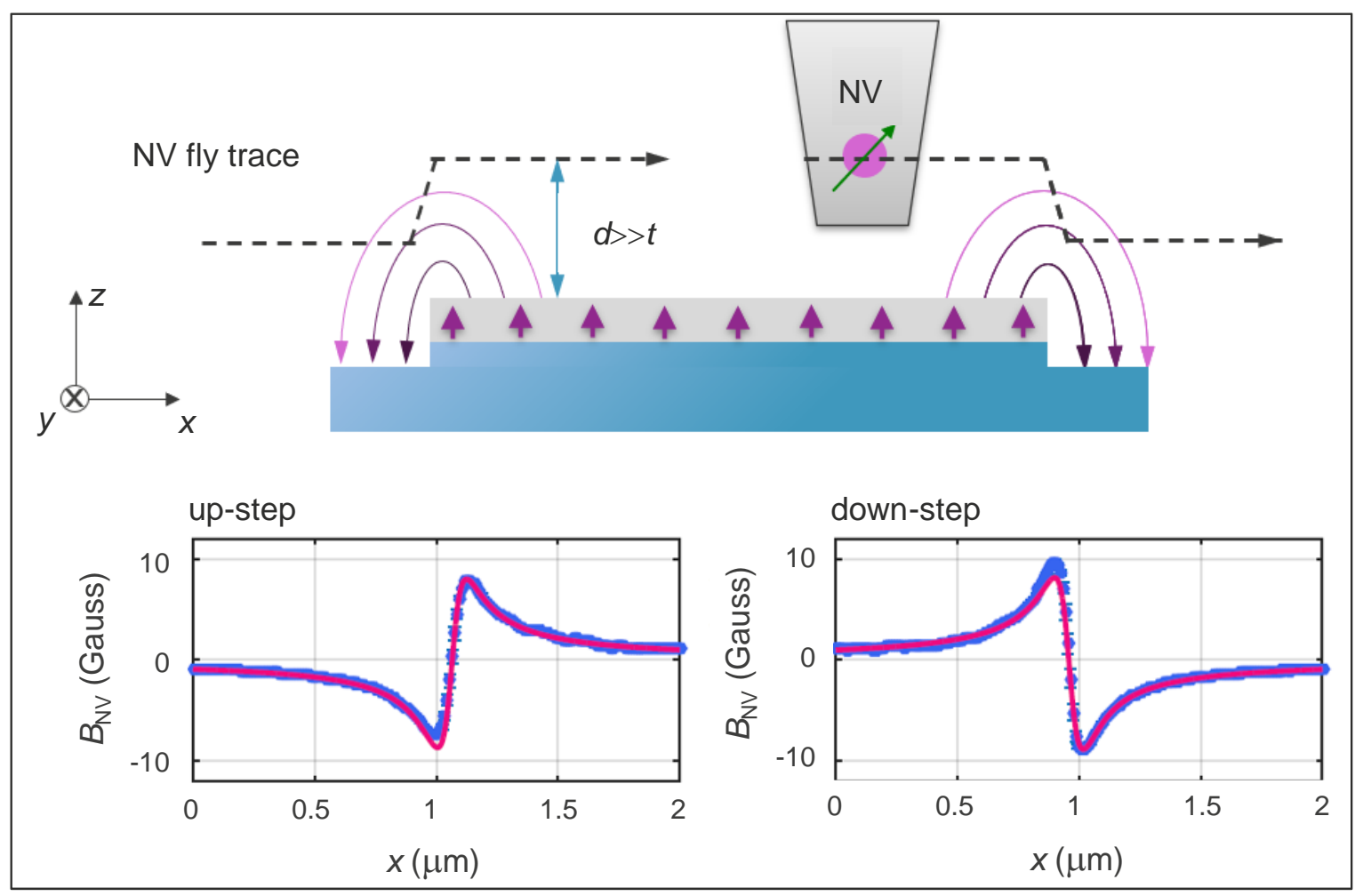

Figure S4. NV-to-sample distance calibration. The single NV defect carried by a pure diamond tip integrated in a quartz tuning-fork sensor, operating in shear force mode, flies at a distance of $d$ above the ultrathin perpendicularly magnetized calibration sample with a thickness of $t$. The calibrations have been done in both up-step and down-step sides of a very wide $\mathrm{CoFeB}$ strip $(20 \mu \mathrm{m}$ wide along $x$ direction, and $200 \mu \mathrm{m}$ long along $y$ direction). The AFM profiles (dash line) of the up- (down-) wards step ( $20 \mathrm{~nm})$ is also taken into consideration in the fitting procedure. The blue scatters with error bars are measured data points while the red solid lines are fitting results. Each data point is integrated for a duration of $\sim 5$ seconds. Due to the longer width of the strip, and thus the extremely long measurement time, only a range of $2 \mu \mathrm{m}$ crosses the step-edges were measured. 
Supporting Information Fig. S5: Simulation of SNVM images (full-B mode and iso-B mode)

The full-B mode SNVM image simulation as shown in Fig. 2 and 3 of the main text, was done in the following procedures:

1. The geometry of CoFeB NWs is modeled according to the TEM morphology results (cf. Fig. S2). The NW is further meshed into unit cells with a size of $(2,5,3) \mathrm{nm}^{3}$ along $x, y$ and $z$ directions.

2. For simplicity, we assume the defective segment in the $x-z$ plane is covering the whole section area of the NWs with a uniformly reduced magnetization of $M=(1-\delta) \cdot M_{\mathrm{s}}$. The nominal value of $M \mathrm{~s}=1200 \mathrm{emu} / \mathrm{cc}$ is used through the whole simulation. The length of each defective segment along the $y$ direction is based on the TEM section results, cf. Fig. S2e, where an average defect size between 10 to $50 \mathrm{~nm}$ can be observed, with a random distribution along the NWs. The magnetization reduction $(\delta)$ in the SNVM image simulations is assumed to be a random value between 0 0.3. In our modeling here, we assume the same random distribution of defect-free segments with a uniform magnetization of $M=M$ s.

3. The stray field of each segment is calculated based on the equation of $\boldsymbol{B}=-N \cdot \boldsymbol{M}$, where $\boldsymbol{B}$ is the vectoral stray field outside each segment, and $\boldsymbol{M}$ is the magnetization of the corresponding segment, and $N$ is the demagnetizing matrix, which is a dimensionless tensor matrix only depending on the structural geometry.

4. A NV-to-sample distance of $50 \mathrm{~nm}$ was used in the simulation. The height $\mathrm{z}$ at which the stray field is 'probed' by the NV (Fig. S5) depends on the position $x$ according to the topographical profile followed by the AFM tip, which resembles a top-hat function. The function $z(x)$ can be expressed by $z(x)=d_{\mathrm{NV}}+\delta z(x)$. The AFM path function $\delta z(\mathrm{x})$ is shown in dash line, extracted from AFM measurements, with an offset such that $\delta z(x)=-h$ stripe $=-45$ $\mathrm{nm}$ when the tip stands on the substrate, and otherwise $\delta z(x)=0$ when the tip stands on the wire.

5. For the two NWs in the simulation, for the regions where $|y|>1 \mathrm{um}$, is the NWs are assumed to be infinitely long uniformly magnetized zones with a magnetization of $M=M \mathrm{~s}$ in order to avoid the edge artifacts in the simulated images. 
6. At each pixel site $\left(x_{\mathrm{i}}, y_{\mathrm{j}}\right)$ at the imaging height $d_{\mathrm{NV}}+\delta \mathrm{z}$, the contributions from all the segments in two NWs are added up and the total vector magnetic fields at this pixel can be calculated.

7. The final quantitative NV image can be calculated considering the projection of the magnetic field along the NV quantization axis using the unitary directional vector $\tau_{\mathrm{NV}}=$ $[\sin \theta \cos \phi, \sin \theta \sin \phi, \cos \theta]$. In the simulation, the NV angles $\left(\theta_{0}, \phi_{0}\right)=\left(55^{\circ}, 270^{\circ}\right)$ are used.

8. From the step 7 , by just taking one step further, the single iso-B mode image can be extracted from the corresponding full- $B$ image. Then for a predetermined $B$ iso field, the corresponding PL signal can be calculated from the $B_{\mathrm{NV}}$ value in each pixel position. By pixel-to-pixel conversion, an iso-B image can be converted directly from the simulated full-B image. 
a)

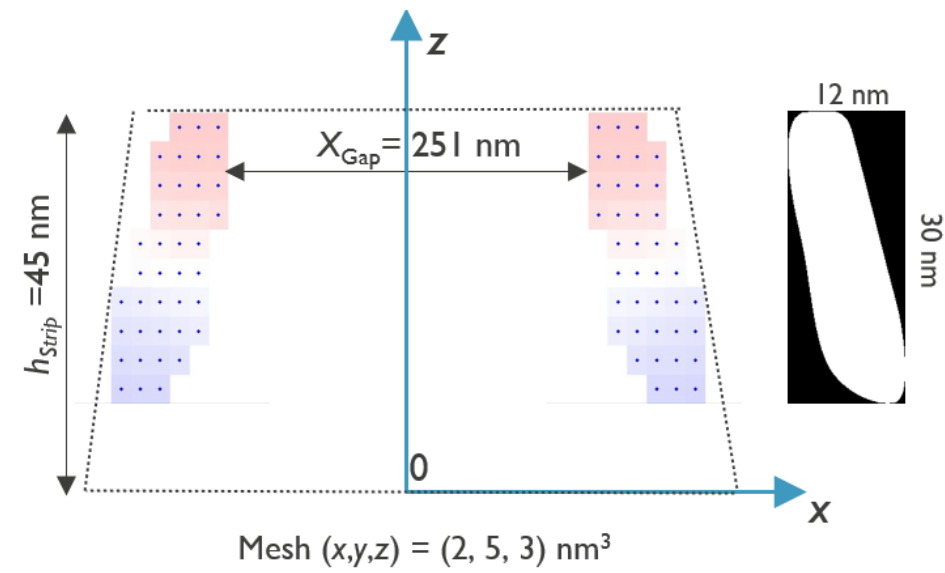

b)

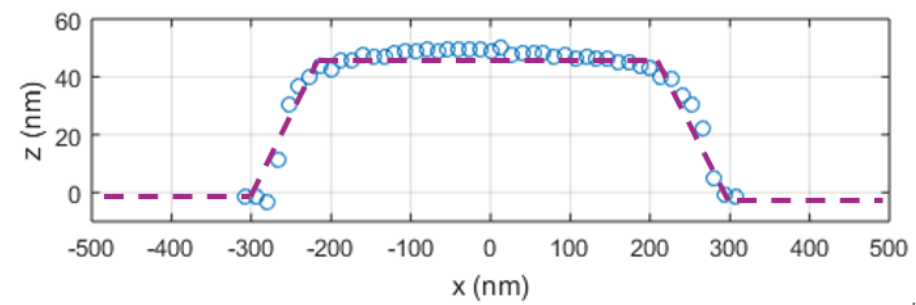

Figure S5. SNVM image simulation mesh model. a, Section view of one NW strip (cf. Fig. $2 \mathrm{c}$ in the main text) structure including the left and right $\mathrm{CoFeB}$ NWs along the $y$ direction. The physical boundary is highlighted by dotted lines. The mesh grid in the section plane is based on the TEM section results depicted in the inset (cf. Fig. 2c in the main text). The mesh unit in the magnetostatic stray field calculation is set to be $(2,5,3)$ $\mathrm{nm}^{3}$ along $x, y$ and $z$ directions. The NW gaps is estimated to be $\sim 251 \mathrm{~nm}$ according to the TEM results, the strip height is measured to be $45 \mathrm{~nm}$, which is confirmed by the AFM profile scan across the two step edges, as shown in $\mathbf{b}$. The physical boarding in the AFM profile (purple dash line), compared to the physical size of the stripe structure as shown in TEM is due to the finite size of the diamond scanning probe with a nominal flat-head tip end diameter of $\sim 200 \mathrm{~nm}$. The physical tip flying path is considered in the SNVM image simulation according to the real experimental AFM scanning process. 
Supporting Information Fig. S6 Micromagnetic simulations

Micromagnetic simulations have been performed using the Object-Oriented Micromagnetic Framework (OOMMF) software package [Cite OOMMF main text]. The simulated geometry is based on the dimensions and shape extracted from a cross section TEM image of a real wire (cf. Fig. S2). Typical magnetic material parameters for $\mathrm{CoFeB}$ were used: the saturation magnetization $\mathrm{M}_{\mathrm{s}}=1.2 \mathrm{MA} / \mathrm{m}$, the exchange stiffness constant $\mathrm{A}=18.6 \mathrm{pJ} / \mathrm{m}$, the Landé factor $g=2.07$, and the Gilbert damping was $a=4 \times 10^{-3}$. The components of the magnetic field extracted in plane situated at $50 \mathrm{~nm}$ height from the wire are shown in Fig. M1 in the presence/absence of a magnetic domain wall in center of the wire.

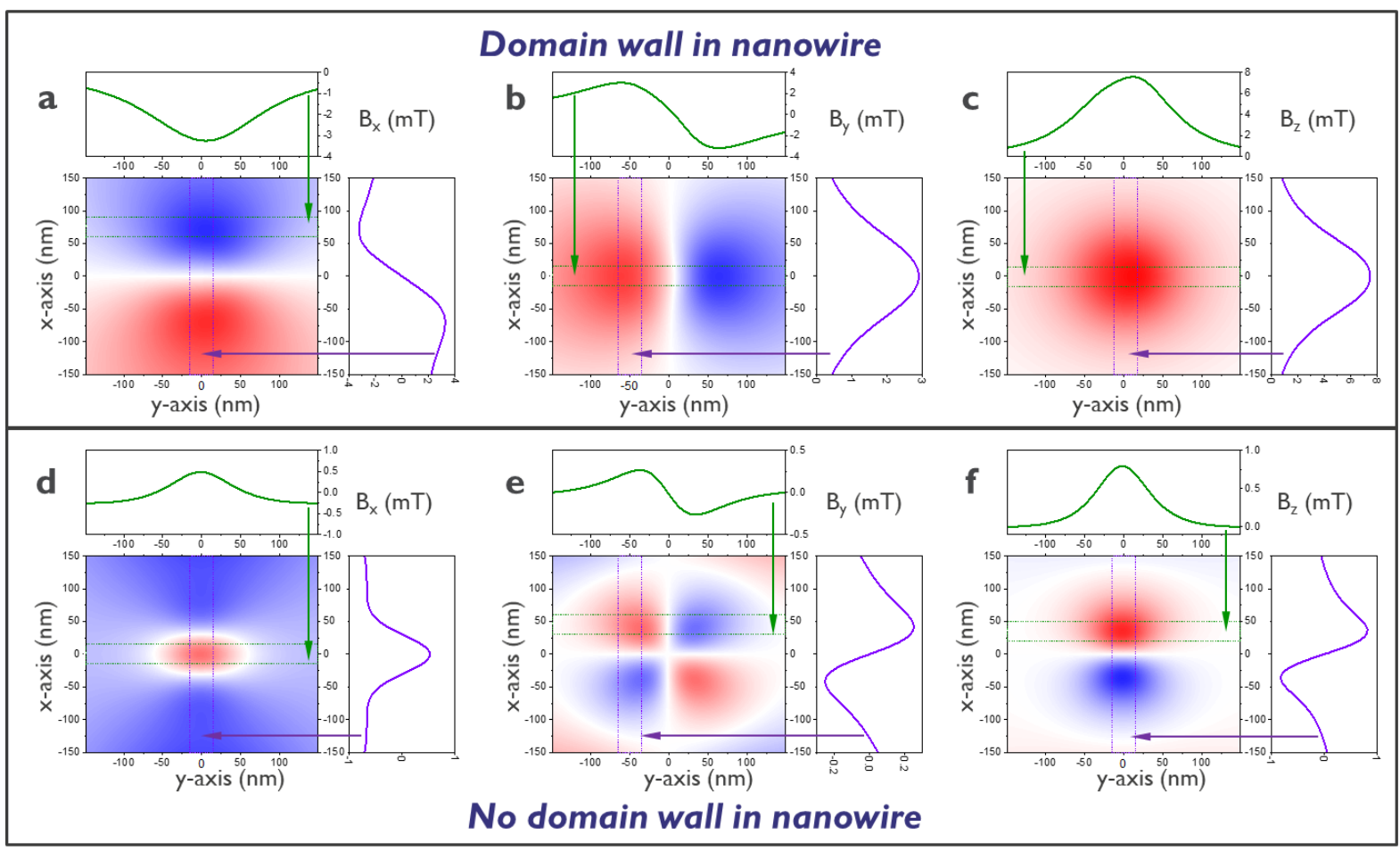

Figure S6. Spatial distributions of the $\mathrm{B}_{\mathrm{x}}, \mathrm{B}_{\mathrm{y}}$ and $\mathrm{B}_{\mathrm{z}}$ components of the magnetic field extracted from micromagnetic simulations of a wire with and without a magnetic domain wall in center of the wire (top and bottom pan. The corresponding magnetic configurations are shown in Fig. 4 of the main text. 


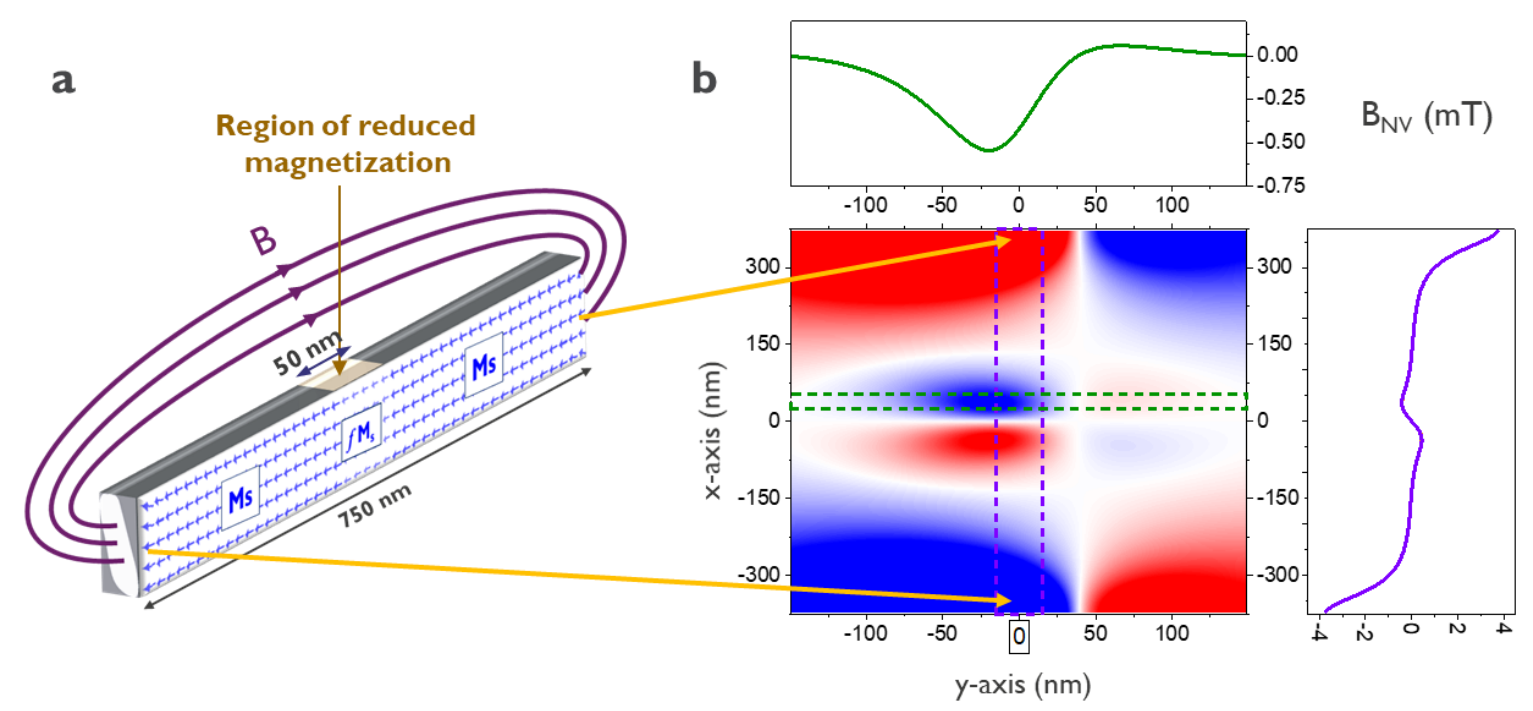

Figure S7. a. Magnetization distribution in a wire with a nonuniformity of reduced magnetization $(f=0.8)$ and a schematic of the magnetic field lines on top of the wire. $b$. Spatial distribution of magnetic field projected on the NV axis extracted in a plane at $50 \mathrm{~nm}$ height from the wire.
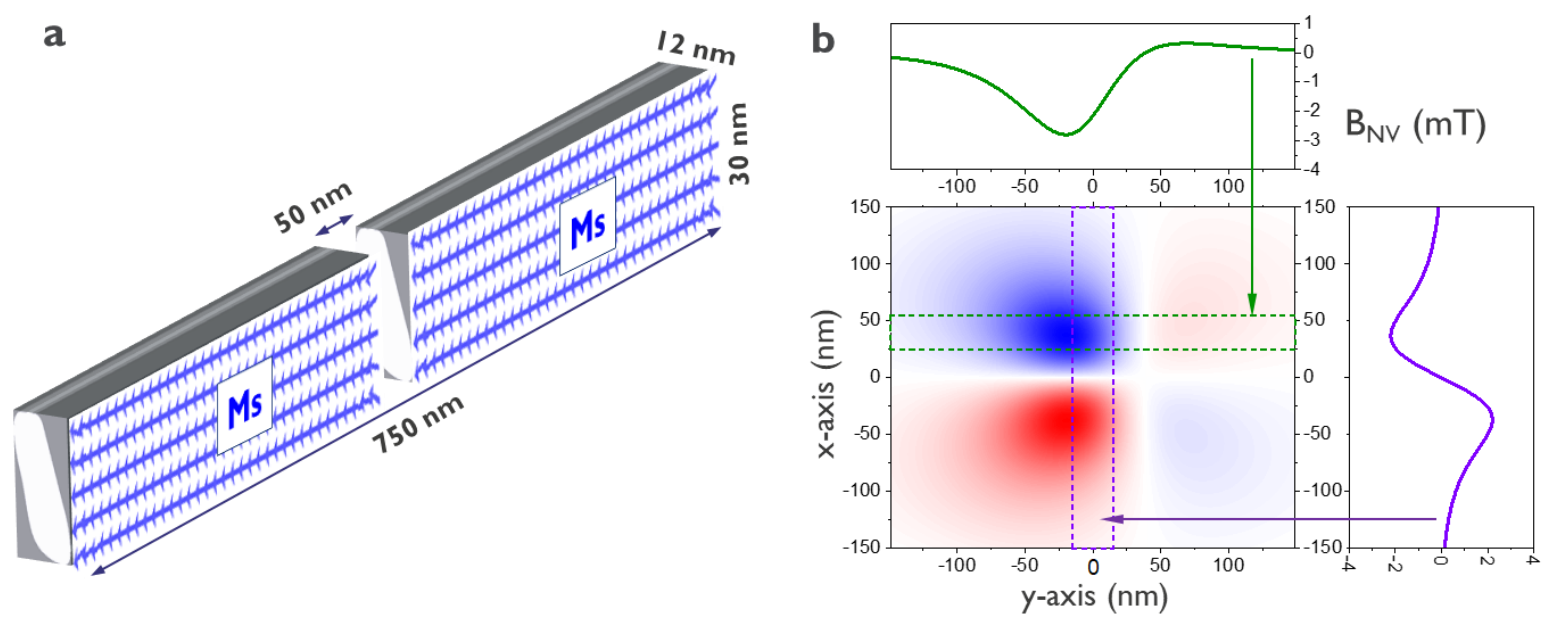

Figure S8. a. Schematic of the simulated wire with a $50 \mathrm{~nm}$ physical gap and the magnetization distribution. b. Spatial distribution of magnetic field projected on the NV axis extracted in a plane at $50 \mathrm{~nm}$ height from the wire. 


\section{References:}

[1]: T. Hingant, et al. Measuring the Magnetic Moment Density in Patterned Ultrathin Ferromagnets with Submicrometer Resolution. Phys. Rev. Applied 4, 014003 (2015). 